\title{
Pathology of spinal cord injuries due to fracture-dislocations of the thoracic and lumbar spine
}

\author{
H Kinoshita \\ Director, Kosei Hospital, Hiroshima, Japan
}

The pathology of intervertebral disc injuries, hyper-extension injuries and spinal cord injuries due to fracture-dislocations of the cervical spine has been reported previously. ${ }^{1-3}$ Between 1957 and 1987, autopsy was performed on 4 patients with fracture-dislocation of the thoracic spine and on one patient with that of the lumbar spine. This paper presents the results of a clinicopathologic study conducted on two patients with a fracture-dislocation of the thoracic spine and on one patient with that pathology of the lumbar spine. The methods employed in the present study are the same as those that were described in my previous report on the pathology of cervical inter-vertebral disc injuries. ${ }^{1}$

Keywords: pathology of spinal cord injuries; fracture-dislocations; thoracic spine; lumbar spine

\section{Case reports}

Case 1

A 50-year-old man was found unconscious resulting from a fall from a height of 3 meters on to rocks in a quarry. At time of his arrival at our hospital by ambulance his heart had stopped and therefore norepinephrine was immediately injected into the ventricle. After resuscitation from apparent death by the application of pressure on to his chest he regained conciousness and survived with respiratory assistance. There were bruises on the face. The right upper extremity was amputated below the shoulder.

The roentgenograms of the thoracic spine revealed a compression fracture with an anterior marginal fragment of the T8 vertebra associated with slight anterior spondylolisthesis of the T7 on the T8 (Figure 1). There was complete paraplegia with anesthesia below the T7 dermatome bilaterally. He died one week after the injury.

Autopsy revealed a compression fracture with a triangular fragment in the anterior superior margin of the T8 vertebra, and fractures of the spinous processes of T7 and 8 vertebrae. There was slight anterior dislocation of the T7 on the T8. Ruptures of all the ligaments and of the intervertebral disc between the T7 and T8 vertebrae, except for the anterior longitudinal ligament were observed (Figure 2). The spinal cord was completely crushed for a distance of $2 \mathrm{~cm}$ by the extruded soft tissues which were destroyed and extruded into the vertebral canal at the T7-8 level. Microscopically the dura was not ruptured but was crushed flat. The spinal cord was completely severed

Correspondence: H Kinoshita, 86-2 Ichiida, Kurose-cho, Kamo-gun, Hiroshima-ken 724-06, Japan

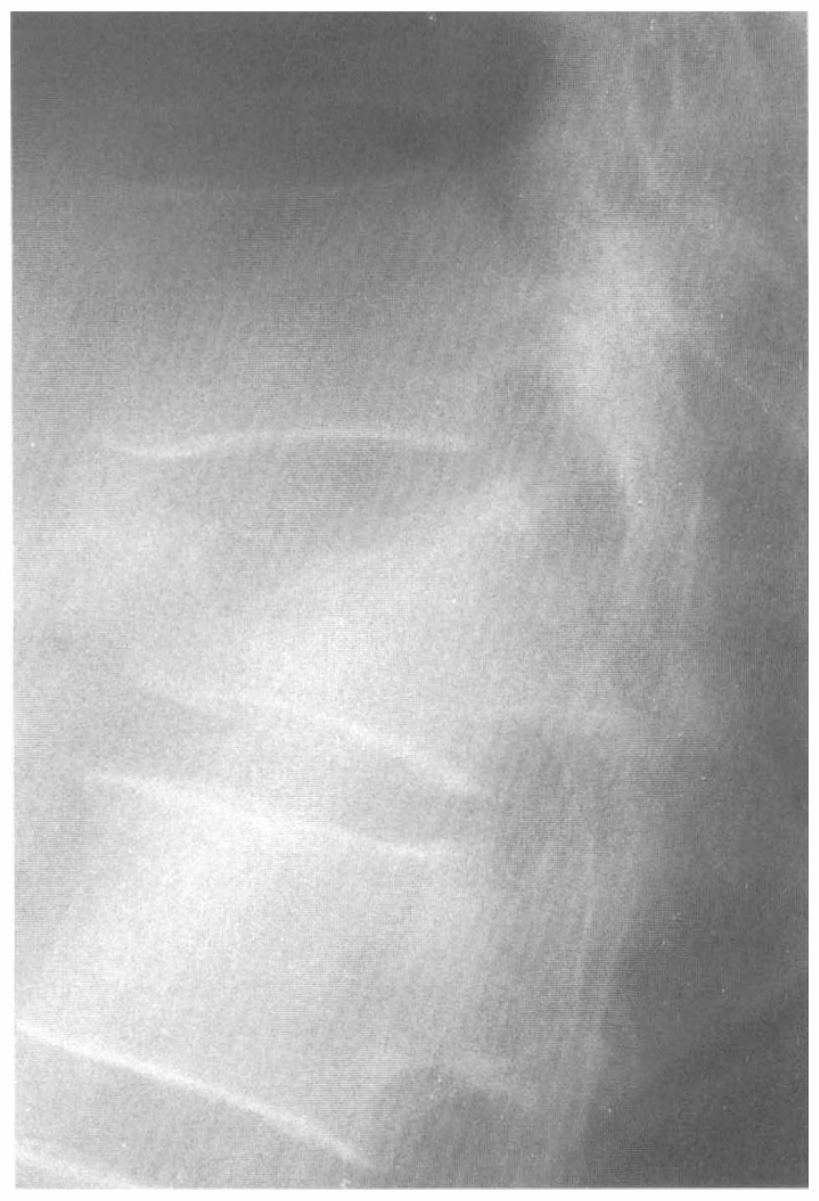

Figure 1 Anterior spondylolisthesis of the T7 on the fractured T8 with anterior marginal fragment 


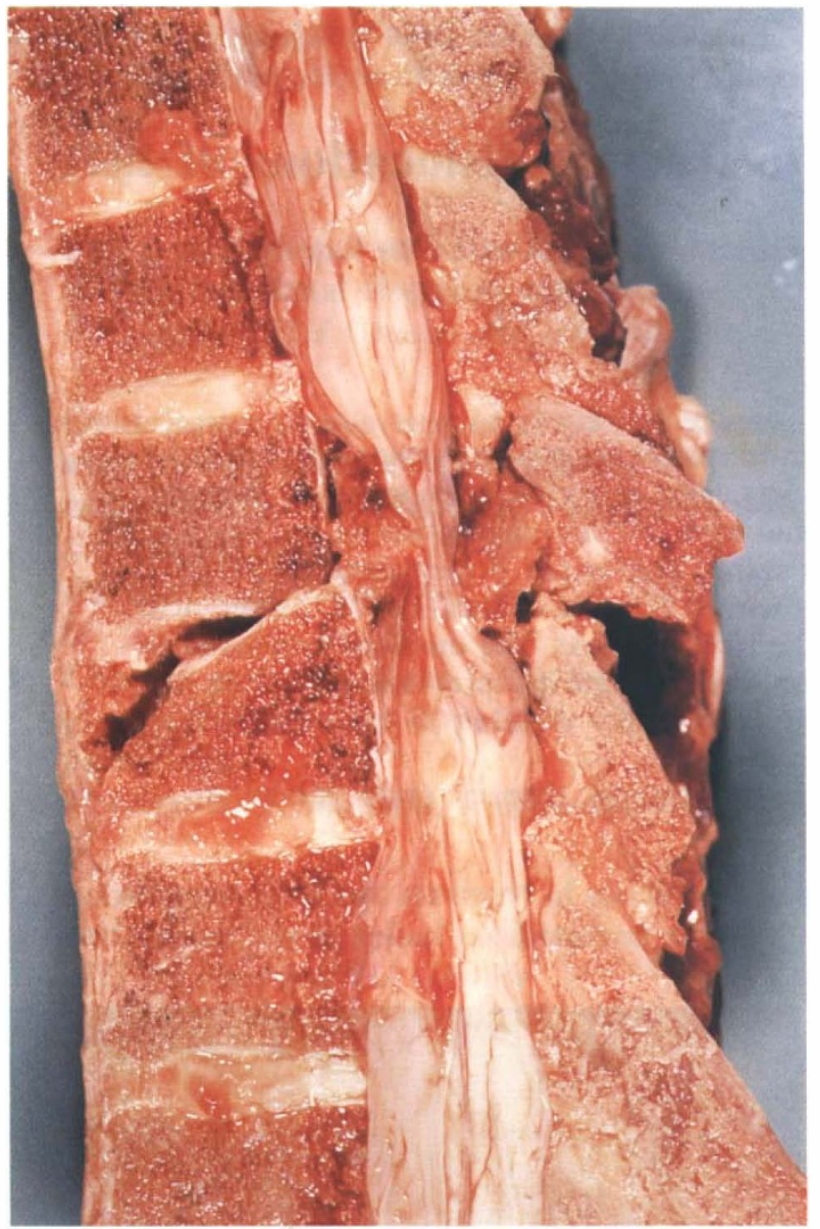

Figure 2 Vertical sagittal section made of the column. Complete disruption of the thoracic spine at T7-8 except the anterior longitudinal ligament

and the cord parenchyma had disappeared. There were small fragments of neural tissue and some fibrin were observed in the subarachnoid space (Figure 3).

\section{Case 2}

A 24-year-old man fell from a telephone pole more than 10 meters in height. His lower extremities were immediately paralyzed with numbness from T10 distally. He was admitted to a local hospital where he was treated by bed rest without changing his body position. On the 30th day after the injury he was transferred to our hospital.

There were large pressure ulcers in the sacral and bilateral fibrocalcaneal regions, and severe edema of both lower extremites. He had complete loss of all sensory modalities below the level of the T10 dermatome, and absence of bladder and bowel control. Both lower extremities were completely paralyzed, and the lower abdominal reflexes and the patellar tendon reflexes were absent. The achilles tendon reflexes were hyperactive, and ankle clonus was demonstrated.

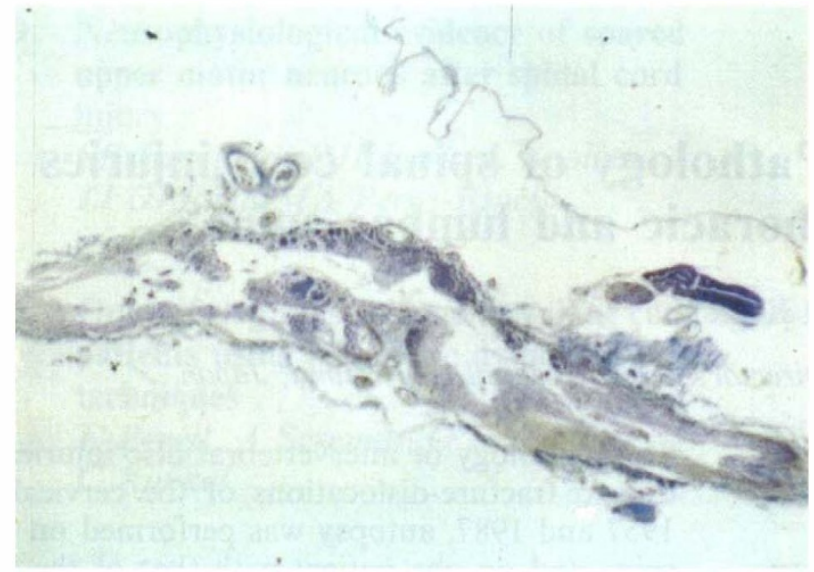

Figure 3 Transverse section at the site of cord lesion, showing disappearance of the parenchyma

Roentgenograms of the thoracic spine revealed a fracture-dislocation of the T8 on the T9 with fracture of the spinous processes of the $\mathrm{T} 7$ and 8 . Anterospondylolisthesis of the T8 vertebra was $40 \%$ of the width of the vertebral body. The patellar tendon reflexes were present 10 weeks after the injury. He had sensation of touch, motion and position in the lower extremities, and some restoration of urinary bladder control. Sitting exercises were commenced at the third month. The intensity of reflex activity increased, and some spasticity was observed in the lower extremities. The patient died from renal failure 157 days after sustaining his injury.

Autopsy was performed: vertical section of the vertebral column with the cord in situ, there was a compression fracture of the T8 vertebra with intervertebral T8-9 disc destruction. Anterospondylolisthesis of the T8 on the T9 was $40 \%$ of the width of the vertebra. The anterior longitudinal ligament was intact and separated from the anterior superior margin of the T9 vertebra, and there was bony self-formation at the site of ligament separation. The neural canal above the site of fracture-dislocation was wide because of the fracture of the inter-articular portion of the T7-8. The spinal cord was constricted and was thin at the site of fracture of T8, without any evidence of crushing (Figure 4). Microscopic study was not made.

\section{Case 3}

A 51-year-old man was crushed by a crankshaft weighing $6000 \mathrm{~kg}$ which dropped from a height of one meter on to his back, and he sustained immediate paralysis and numbness of the lower extremeties. He was taken to a local hospital where a plaster cast fixation of the trunk was applied.

The patient was transferred to our hospital 5 days after injury. Severe edema of both lower extremities was observed, together with large decubitus ulcers of the sacral and bilateral fibrocalcaneal regions. He had 
a level of hypaesthesia to the inguinal region on the right side and L2 on the left side. There was complete loss of sensation below S2 symmetrically. He could reasonably move his thighs and legs, but the power of extension and of abduction of the thighs and of flexion of his legs were weak. There was no voluntary movement of his ankles and toes. There was a patellar tendon reflex on the right side; absent on the left. The achilles tendon reflexes were absent. The cremaster reflex was present on the right side, but absent on the left. The anal skin and bulbocaverous reflexes were absent. There was incontinence of feces and of urine. It was thus necessary to insert an indwelling catheter into the urinary bladder.

Roentgenograms of the lumbar spine showed that the left half of the vetebral body of L4 was severely crushed, with fracture of the right upper articular process of L5 and of the left upper articular process of L4 and of the transverse processes bilaterally. L4 verterbra was displaced posterolaterally to the right

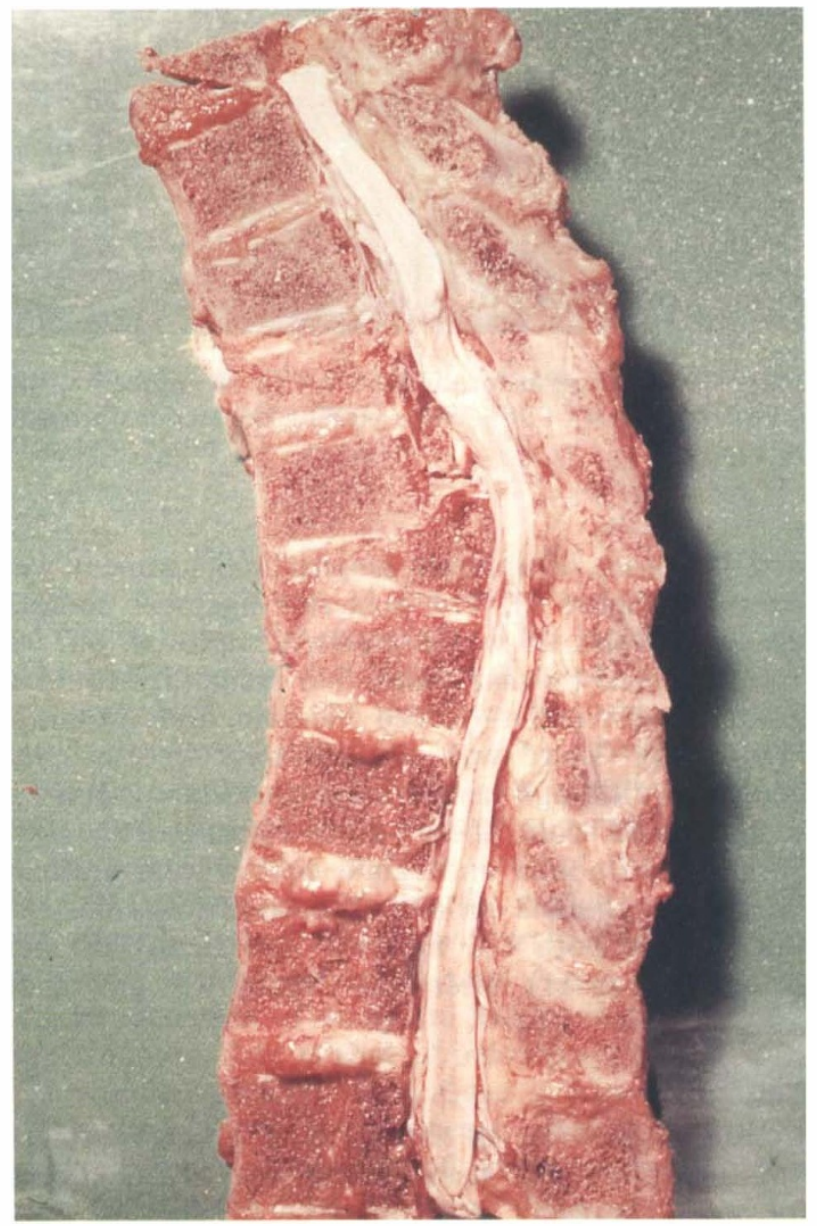

Figure 4 Vertical section of the thoracic column with the spinal cord in situ. Anterior spondylolisthesis of the T8 on the fractured T9. Fractures of the interarticular portion of the T7 and 8 produced a lengthening of the neural canal and the spinal cord escaped crushing and inferiorly on L5 vetebra. The left L4-5 upper articulation was disengaged. The right half of the L4 vertebral body remained intact (Figures 5 and 6).

Two weeks after the injury, open operative reduction was carried out with the patient in the prone position, and with the head and lower extremities placed in a sling attached to the ankles for hyperextension of the spine. The site of the injury was exposed. The interspinous ligaments were seen to be ruptured, without fracture of the lamina, but the dura was intact. The L4 vertebra was displaced to the left posterolaterally on L5.

Hooks were placed on the intact spinous processes of L3 and L5. While applying traction to the spine the dislocated L4 vertebra was carefully reduced on L 5 by two hooks. The patient's head was then fixed in position and the extremities were drawn upwards by means of a sling and pulley. With hyper-extension of the spine the kyphotic angulation was corrected and fracture of the vertebral body was reduced. Transverse holes were made through the base of the spinous processes of L3, 4 and 5. Soft stainless steel wires $1 \mathrm{~mm}$ in diameter were placed through these holes and looped as is shown in Figure 8.

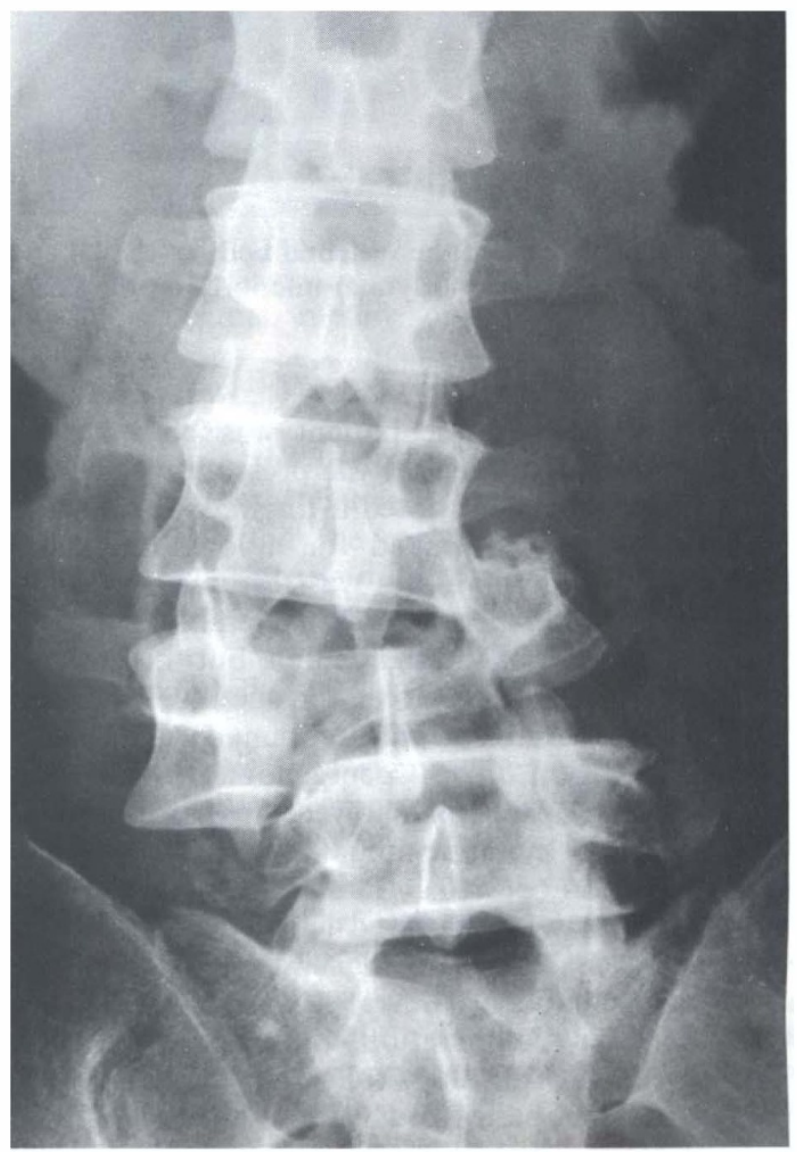

Figure 5 Fracture-dislocation of the L4 right laterally and inferiorly on the L5. Left upper articular process of the L4 and right upper articular process of the L5 are fractured 


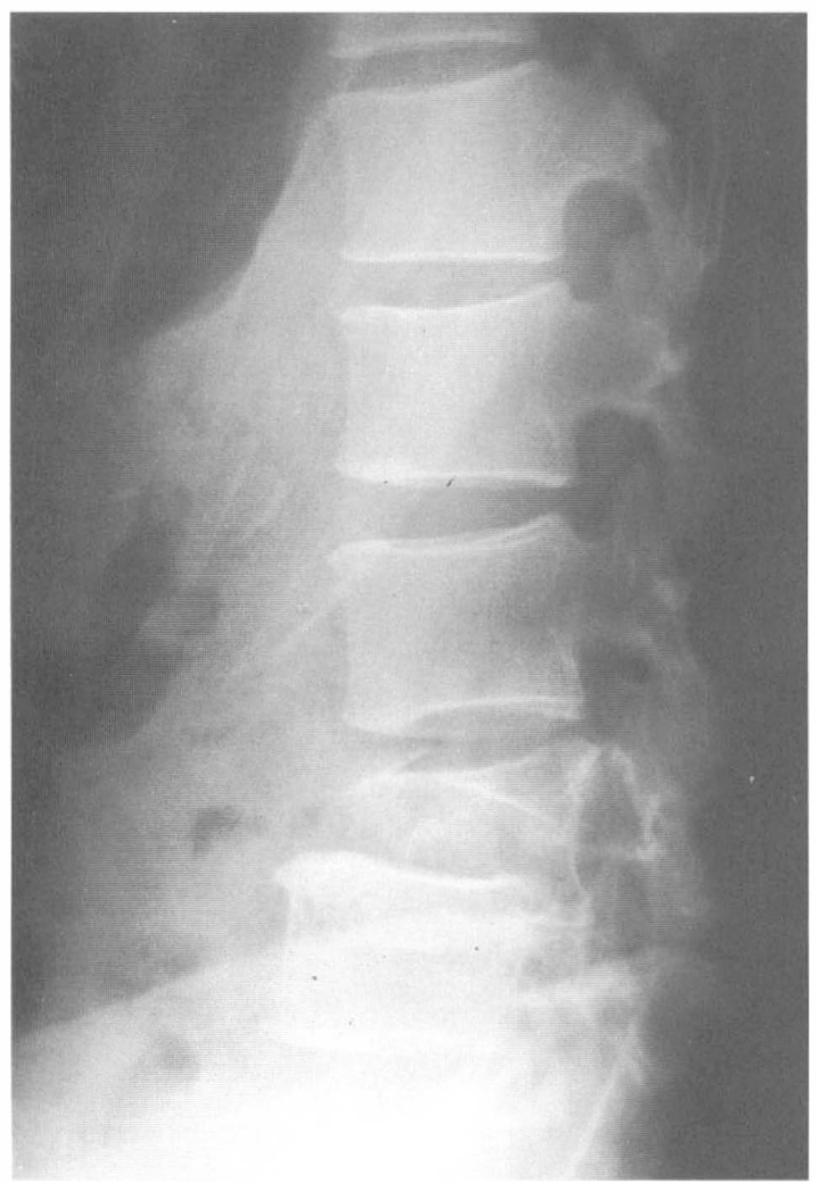

Figure 6 Severe fracture of the vertebral body of the L4 and dislocation of the L4 posteriorly and inferiorly on the L5

After the operation, the patient was placed on a Stryker frame for treatment of the pressure ulcers. The following day the patient noted a tingling sensation in his right leg which continued for a long time, and sensation in S2, 3 dermatomes was restored. Ten days after the operation, there was considerable improvement in the strength of the right thigh, but the findings in the left thigh and in both feet and the toes remained unchanged. Bed exercises were commenced 4 weeks after the operation. The roentgenograms taken 5 weeks after the operation showed neither posterior displacement nor kyphotic angulation. There was a lateral angulation of $16^{\circ}$. The wires binding the spinous processes were ruptured (Figure 7).

Recovery of neurological deficit was expected in this situation but he suddenly developed a hemorrhage on the 37 th day after the operation, and died 2 days later. $\mathrm{He}$ had survived for 53 days following the accident.

An autopsy was performed: lateral roentgenograms of the resected lumbar spine showed anatomical reduction of the fracture-dislocation of the L4-5 (Figure 8). Macroscopically, neither dislocation nor deformity were observed. Fracture of the transverse processes of left L2, right L3, bilateral L4 and L5 and

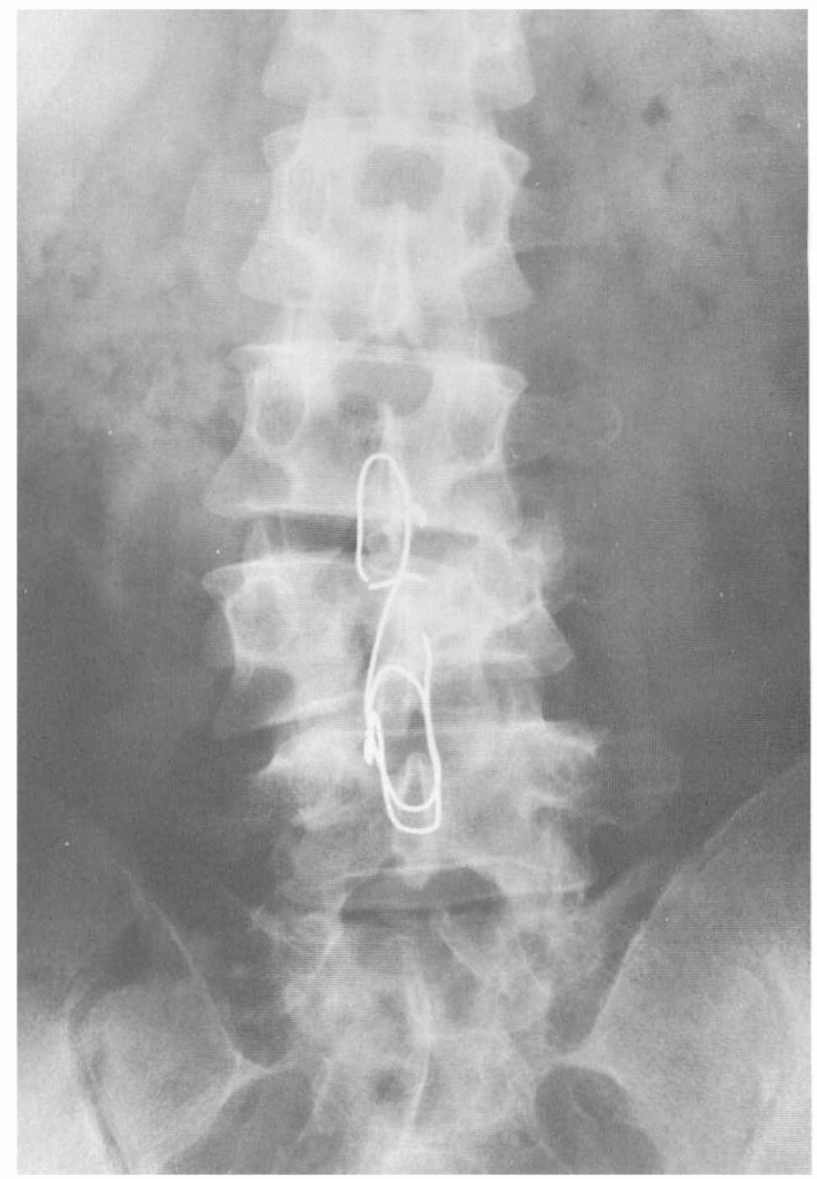

Figure 7 Fracture-dislocation of L4-5 is reduced with a lateral angulation of $16^{\circ}$. Wires binding the spinous processes are ruptured

partial rupture of anterior longitudinal ligament were observed (Figure 9). The posterior ligament complex between L4 and L5 was completely ruptured, but was firmly adhered by scar tissue. No instability could be demonstrated under forced flexion and extension (Figure 10). There was no deformity in the spinal canal, and although hemorrhage could be observed in the extradural space, there was no rupture of the dura. The pia-arachnoidia and cauda equina were mutually adhered for about $2 \mathrm{~cm}$ and separation was somewhat difficult, but both the firmness and the color were normal (Figure 11). Microscopy study was not made.

\section{Discussion}

Fracture-dislocation of the thoracic spine associated with paraplegia is usually the result of extreme violence. ${ }^{4}$ The exact mechanism of the thoracic spine injury in Case 1 could not be ascertained as there was no one present at the accident site. The postmortem study showed complete disruption of the thoracic spine except for the anterior longitudinal ligament. These findings suggest that the lower dorsal spine had been struck obliquely downwards and forwards by a force 


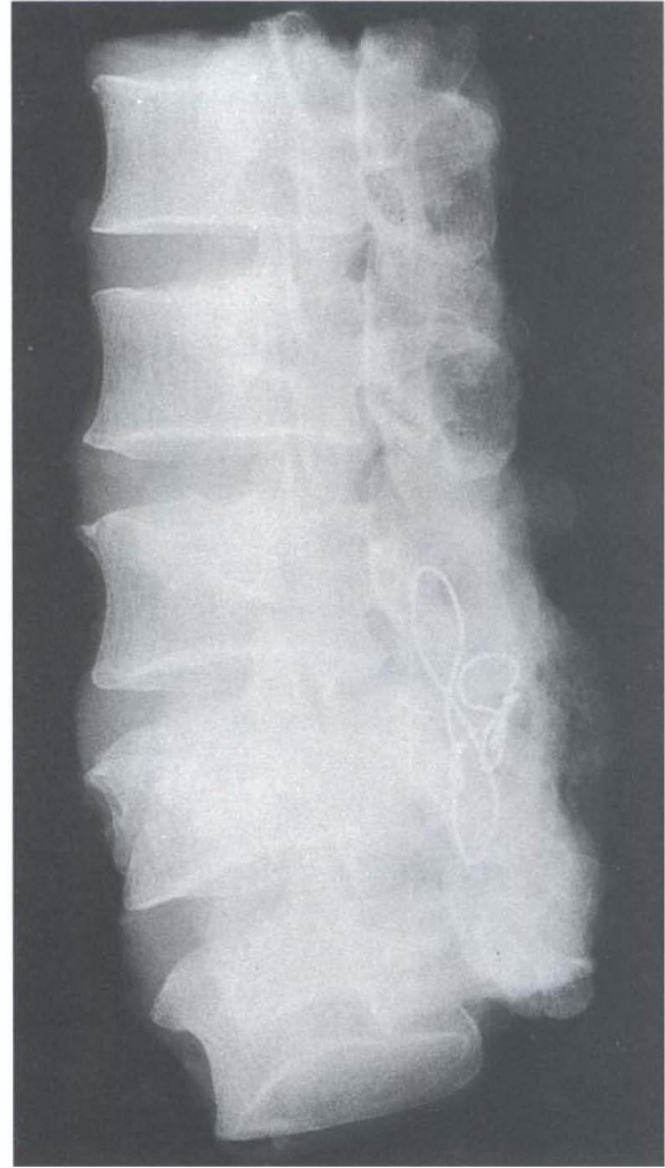

Figure 8 Lateral roentgenograms of the resected lumbar spine, showing anatomical reduction of the fracture dislocation of the L4-5

acting in the direction of the intervertebral disc, and the T8 vertebra slid forwards. The violence of the injury was so great that there was total disruption of the spinal cord with the vertebral fracture. Generally flexion fracture-dislocation of the thoracic spine narrowing of the neural canal and crushing of the spinal cord between the posterior superior margin of the fractured vertebra and the arch of the forwardly displaced vetebra occurs. Hardy and Rossier (1975) have described that clinical asymmetry of lesions in those with a thoracic spinal cord injury is rare, and that the incidence of complete lesions is twenty times more common than is that of incomplete lesions. ${ }^{4}$

The patient described as Case 2 had a complete neurological lesion, but 10 weeks after the injury he recovered some modalities of sensation, and partial restoration of bladder function. Autopsy showed a fracture through the interarticular portion of the T7, 8 vertebrae and diastasis between the vertebral bodies and the spinal processes. The T7, 8 vertebral body with fractured laminae slipped forward after the rupture of the T8-9 intervertebral disc, as in spondylolisthesis. This produces lengthening of the

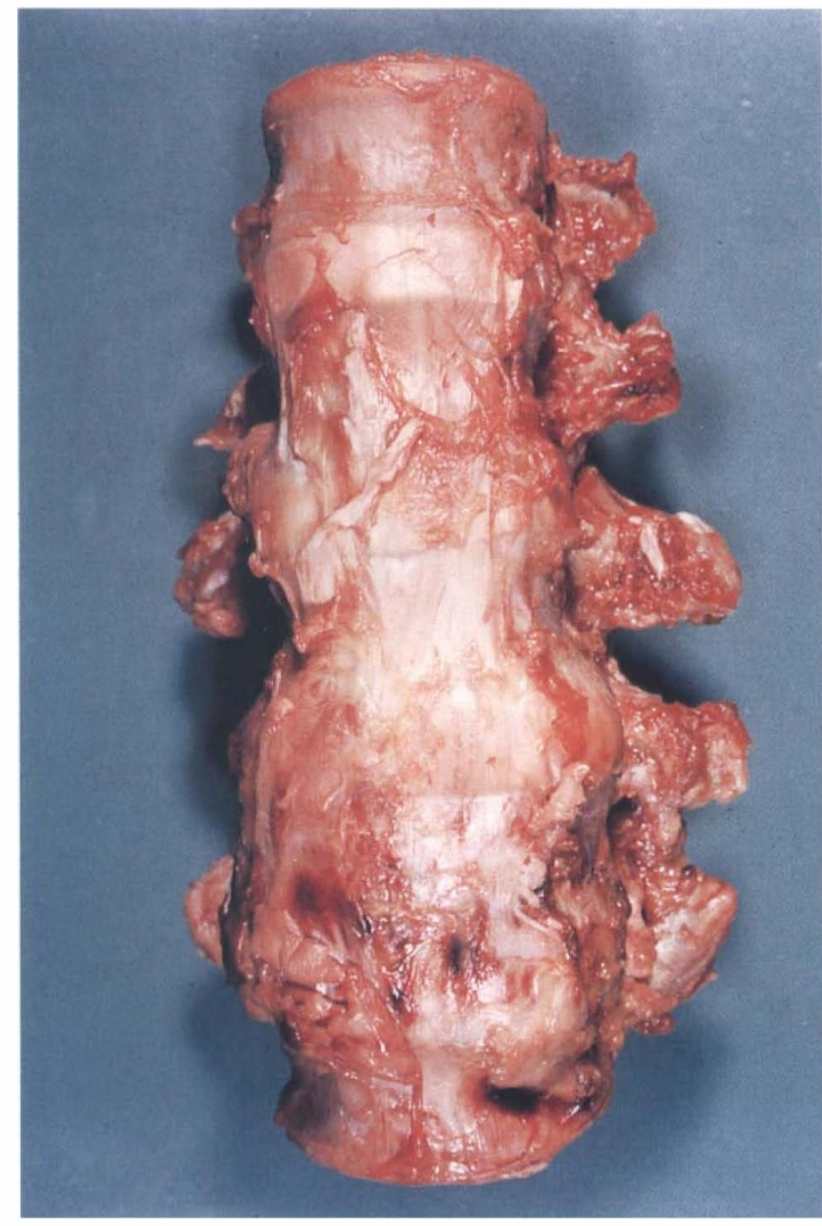

Figure 9 Anterior view of the resected lumbar spine, showing a partial rupture of the anterior longitudinal ligament

neural canal in the sagittal plane and the spinal cord escaped crushing between the posterior superior margin of $\mathrm{T} 9$ and the arch of T8. The spinal cord was constricted at the point of the fractured vertebra. These findings clarified the reason why the patient recovered from a complete lesion despite the $40 \%$ anterospondylolisthesis of T8. Böhler ${ }^{5}$ emphasized that vertebral arch fractures of the spondylotic or spondylolisthetic type signify the maintenance of the spinal cord. Bailey reported a surprising case with roentgenograms showing the body of C5 lying directly in front of $\mathrm{C} 6$, but the patient was able to walk with the aid of a cane. The roentgenograms suggested that she had a vertebral arch fracture of the C4, C5 and her fracture-dislocation was a type of spondylolisthesis.

The fracture-dislocation in Case 3 occurred as a result of vertical compression and torsional violence. The external force acting directly on the lower lumbar spine was so massive that the vertebral body was severely crushed, the interspinous ligaments were ruptured, and the upper articular processes of the L4 and L5 were fractured by the lateral movement produced by the twisting, which permitted the L4 to 


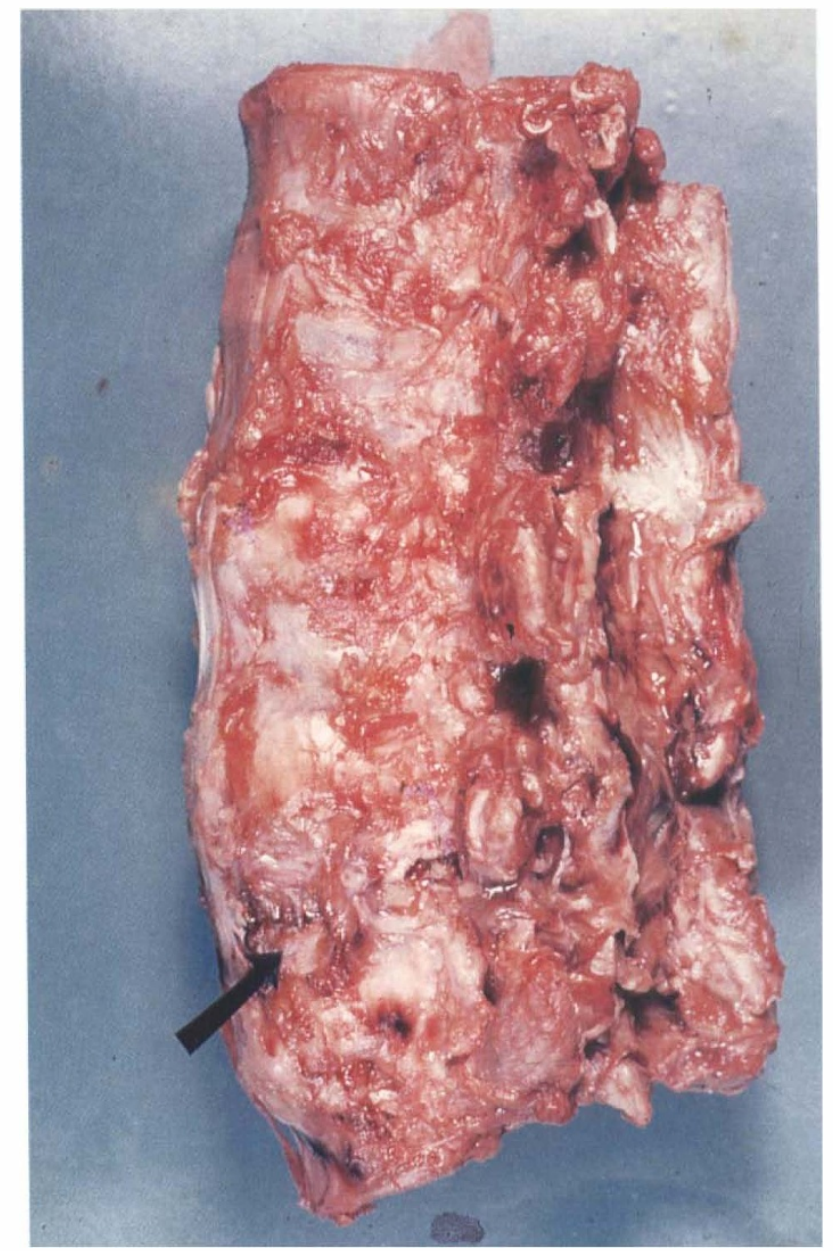

Figure 10 Lateral view of the resected lumbar spine, showing adhesion of the ruptured soft tissue around the intervertebral space and posterior ligament complex of the L4- 5

swing upon the L5 vertebra. Autopsy demonstrated a good anatomical reduction of the fracture-dislocation of the L4-5 and stable fixation of the spine by fibrous adhesions.

During the past 40 years Kinoshita et $a l^{7}$ have treated thoracolumbar and lumbar fracture-dislocations by open reduction of the dislocated facets, correction of fractures of the ventral body by ventral suspension and fixation of the spinal column by wiring the spinous processes. In a follow-up study of 30 patients for more than 5 years, it was observed in 8 patients that the wire had broken from 8.5 weeks to 10 months after the operation, but there was no recurrent case of displacement because of the broken wire. In this case, it was found that the wire had broken 5 weeks after the operation, but the resected lumbar column remained stable due to fibrous adhesions. More rigid stability can be expeccted without recurrence of displacement after a few weeks of bed exercise.

Very few reports have been published in the

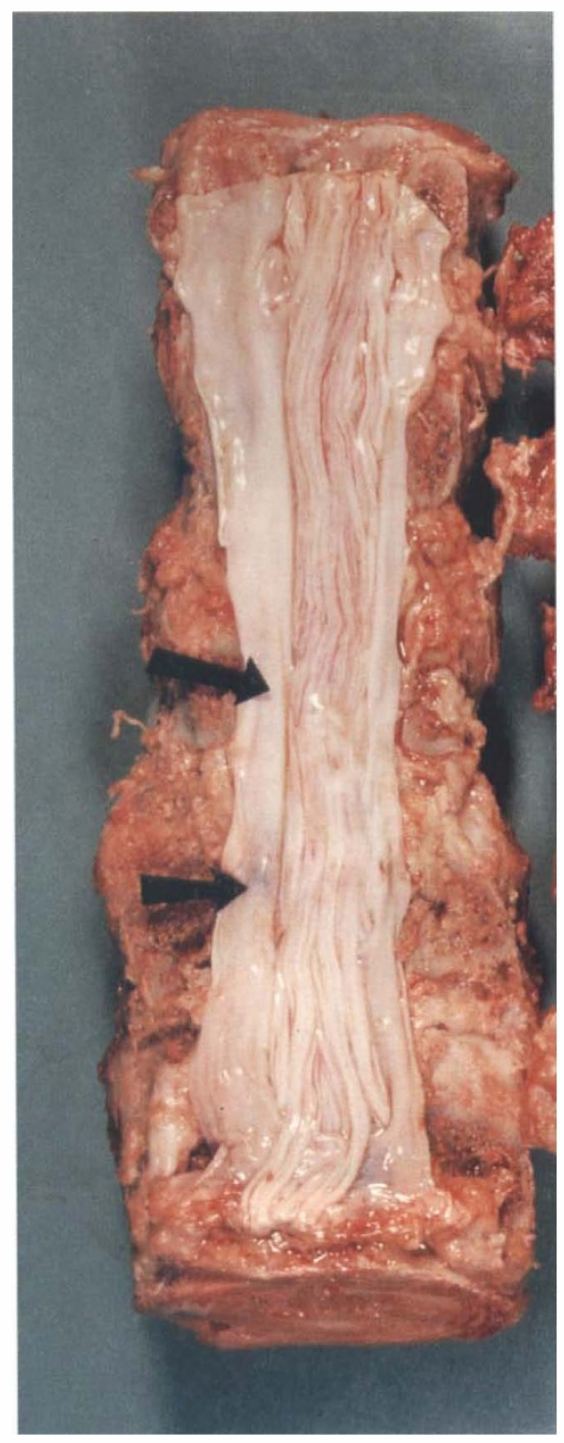

Figure 11 No severance of the cauda equina. Injured cauda equina are adhered to each other. Cauda equina and dura are adhered on the left side

literature on the pathological findings of those with cauda equina lesions. Injury to the cauda equina in this case was not very severe because of the fracture of the left upper articular process of the L4. Though there was no severance of the cauda equina, the left side was more severely injured than was the right side. Clinical features also indicated that the neurological motor deficits of the left lower extremity were more severe than those of the right. Whitesides ${ }^{8}$ stated that injury to the nerve root may be either neurapraxia and axonotomesis or neurotmesis in nature. Pathological findings of the cauda equina of this case revealed that some nerve roots were axonotomesis in nature, and therefore improvement of the paralysis could be expected if he had survived for a long period. 


\section{References}

1 Kinoshita H. Pathology of cervical intervertebral disc injuries. Paraplegia 1993; 31: 553. 559.

2 Kinoshita H. Pathology of hyperextension injuries of the cervical spine. Paraplegia 1994; 32: 367 - 374.

3 Kinoshita $\mathrm{H}$. Pathology of spinal cord injuries due to fracture and fracture-dislocations of the cervical spine. Paraplegia 1994; 32: $642-650$.

4 Hardy AG, Rossier AB. Spinal cord injuries. 1975; George Thieme Publishers, Stuttgart: 61-68.
5 Böhler L. The treatment of fractures. 1956; Grune \& Stratton, New York and London, Volume 1: $311-319$

6 Bailey IC. Fracture dislocation of the cervical spine with gross displacement. 1975; Case Report. J Neurosurg 42: 209-211.

7 Kinoshita H, Nagata Y, Hirakawa H. Thoracolumbar fracture dislocation: A study of 30 patients. Paraplegia 1989; 27: 289295 .

8 Whitesides TE. Traumatic kyphosis of the thoraco-lumbar spine. Clin Orthop Rel Res 1977; 128: $78-92$. 